\title{
A Neurochemically Distinct Dorsal Raphe-Limbic Circuit with a Potential Role in Affective Disorders
}

\author{
Kathryn G Commons*,', K Ryan Connolley' and Rita J Valentino' \\ 'The Children's Hospital of Philadelphia, Abramson Pediatric Research Center, Philadelphia, PA, USA
}

\begin{abstract}
The serotonergic system arising from the dorsal raphe nucleus (DR) has long been implicated in psychiatric disorders, and is considered one site of action of classical anxiolytic and antidepressant agents. Recent studies implicate the DR as a site of action of novel anxiolytic and antidepressant agents that target neuropeptide systems, such as corticotropin-releasing factor (CRF) and neurokinin I (NKI) antagonists. The present study identified unique characteristics of the dorsomedial DR that implicate this particular subregion as a key component of a circuit, which may be targeted by these diverse psychotherapeutic agents. First, it was observed that a cluster of CRFcontaining cell bodies was present in the dorsomedial DR of colchicine-treated rats. Dual-labeling immunohistochemistry revealed that almost all CRF-containing neurons were serotonergic, implicating CRF as a cotransmitter with serotonin in this subpopulation of DR neurons. Moreover, dendrites laden with immunoreactivity for NKI had a striking topographic distribution surrounding and extending into the dorsomedial subregion of the DR, suggesting that NKI receptor ligands may selectively impact the dorsomedial DR. Finally, anterograde tract tracing from the dorsomedial DR combined with CRF immunohistochemistry revealed that CRF-containing axons from this subregion project to CRF-containing neurons of the central nucleus of the amygdala. Taken together, the present results reveal a circuit whereby NKI receptor activation in the dorsomedial DR can impact on limbic sources of CRF that have been implicated in emotional responses. This circuit may be relevant for understanding the mechanism of action of novel psychotherapeutic agents that act through NKI or CRF receptors.

Neuropsychopharmacology (2003) 28, 206-215. doi: I 0.1 038/sj.npp. 1300045
\end{abstract}

Keywords: corticotropin-releasing factor; neurokinin I; serotonin; amygdala; substance P; dorsal raphe nucleus

\section{INTRODUCTION}

Clinical findings have long implicated serotonin in the pathophysiology of affective disorders. For example, levels of serotonin and its major metabolite are decreased in the cerebrospinal fluid of depressed patients and in brain tissue of depressed suicide victims (Nordstrom and Asberg, 1992; van Praag, 1984). Depressed patients also demonstrate reduced responsiveness to a number of pharmacological challenges with serotonin agonists (Cowen, 1993; Lesch, 1991; Mann et al, 1996; Shapira et al, 1992). A role for serotonin in depression and other affective disorders is further underscored by the fact that the most widely used pharmacological treatments for these disorders alter serotonin neurotransmission.

As the dorsal raphe nucleus (DR) is a major source of serotonin innervation of forebrain and limbic regions (Jacobs and Azmitia, 1992; Molliver, 1987), this is a

*Correspondence: KG Commons, The Children's Hospital of Philadelphia, 402 Abramson Pediatric Research Center, 34th and Civic Center Blvd., Philadelphia, PA 19104, USA, Tel: +215 590 0653, Fax: +2I5 590-2759, E-mail: commons@email.chop.edu

Received 22 March 2002; revised 19 June 2002; accepted 2 August 2002

Online publication: 9 August 2002 at http://www.acnp.org/citations/ Npp080902364 potential site of origin of serotonin dysfunction in affective disorders. Consistent with this, substantial anatomical differences were documented in serotonin markers within the DR of depressed suicide subjects vs controls (Arango et $a l, 2001)$. However, the $\mathrm{DR}$ is a complex, heterogeneous nucleus composed of specific clusters of neurons or subnuclei that are morphologically distinct (Jacobs and Azmitia, 1992; Molliver, 1987; O'Hearn and Molliver, 1984; Peyron et al, 1996; Peyron et al, 1998). These subnuclei also differ in their neural connectivity suggesting that they subserve distinct functions. Given this, it is possible that specific subnuclei within the DR are involved in the pathophysiology of affective disorders or are selectively targeted by agents effective in treating these disorders.

A novel approach in the treatment of depression has focused on two neuropeptide systems, the corticotropinreleasing factor (CRF) system and the neurokinin system. Antagonists of the CRF-R1 receptor and the neurokinin 1 (NK1) receptor have reported antidepressant potential in humans (Argyropoulos and Nutt, 2000; Zobel et al, 2000). Basic studies in rodents indicate that these receptors also modulate serotonin neurotransmission (Kirby et al, 2000; Lowry et al, 2000; Price and Lucki, 2001; Santarelli et al, 2001), suggesting that this is a potential mechanism for their clinical efficacy. Evidence for CRF terminal appositions on serotonin neurons in the DR of human brains 
supports this view (Ruggiero et al, 1999). The present study used triple-label immunohistochemistry to identify areas within the DR that may be points of intersection of NK1, $\mathrm{CRF}$, and serotonin, and may explain the common clinical effects of these diverse pharmacological agents. Anterograde tract tracing was used to further elucidate the neural circuits that may be affected by psychotherapeutic agents that target these points of intersection.

\section{MATERIALS AND METHODS}

\section{Animals}

The subjects were 20 adult male Sprague-Dawley rats (Taconic Farms, Germantown, NY) weighing 250-300 g. Rats were housed two to three per cage on a 12-h light schedule (lights on at 07:00) in a temperature controlled $\left(20^{\circ} \mathrm{C}\right)$ colony room and were given free access to standard rat chow and water. Animal protocols were approved by the Institutional Animal Care and Use Committee and were conducted in accordance with the NIH Guide for the Care and Use of Laboratory Animals (1996).

\section{Surgery}

For intraventricular injection of colchicine, rats were anesthetized with halothane and positioned in a stereotaxic apparatus with the snout lowered to place the skull at a $15^{\circ}$ angle from the horizontal. The temperature was monitored throughout the surgical procedure and maintained at $37^{\circ} \mathrm{C}$ using a feedback-controlled heating device. A scalp incision was made and a hole was drilled centered at $1.0 \mathrm{~mm}$ caudal to bregma $1.5 \mathrm{~mm}$ lateral to the midline. Colchicine $(100 \mu \mathrm{g}$ in $5 \mu \mathrm{l}$ saline, i.c.v.; Sigma Chemical, St Louis, MO) was administered through a 26-gauge stainless-steel cannula centered at the above coordinates and lowered $5.6 \mathrm{~mm}$ from the skull surface such that the tip was in the lateral ventricle. The cannula was removed $5 \mathrm{~min}$ later and the cut sutured. Rats were anesthetized with pentobarbital (100 mg/kg, i.p.) and transcardially perfused $24 \mathrm{~h}$ later.

For injection of the anterograde tract tracer, Phaseolus vulgaris leucoagglutinin (Pha-L, Vector Laboratories, Burlingame, CA), rats were surgically prepared as described above with the exception that a hole was drilled to expose the saggital and superficial transverse sinuses. The saggital sinus was ligated and reflected to allow for a midline approach to the DR with minimal blood loss. Glass micropipettes $(20 \mu \mathrm{m}$ diameter tip) filled with Pha-L $(2.5 \%$ in $0.01 \mathrm{M}$ phosphate-buffered saline) were positioned at the midline, $1.8 \mathrm{~mm}$ caudal to the intersection of lambda and midline, and $5 \mathrm{~mm}$ ventral to brain surface. Pha-L was iontophoresed using a current of $5 \mu \mathrm{A}$ ( $7 \mathrm{~s}$ pulses, $50 \%$ duty cycle, $20 \mathrm{~min}$ ). The pipette was left in place for $10 \mathrm{~min}$ to limit leakage of the tracer along the pipette track. The cut was sutured and rats were anesthetized with pentobarbital $(100 \mathrm{mg} / \mathrm{kg}$, i.p.) and transcardially perfused 10 days following the surgery.

\section{Histology}

Rats were transcardially perfused with $50 \mathrm{ml}$ of heparinized saline followed by $300-400 \mathrm{ml}$ of $4 \%$ paraformaldehyde in
0.1 M phosphate buffer (PB). Brains were removed, cut into $3 \mathrm{~mm}$ coronal blocks and stored in the paraformaldehyde solution for $2-24 \mathrm{~h}$, then equilibrated in $20-30 \%$ sucrose solution. The blocks were rapidly frozen and $40 \mu \mathrm{m}$ sections were cut on a cryostat. Sections were collected in $0.1 \mathrm{M} \mathrm{PB}$ and incubated in $1 \%$ hydrogen peroxide for $30 \mathrm{~min}$ to quench endogenous peroxidase activity before immunohistochemical processing. All antisera were diluted in $\mathrm{PB}$ with $0.9 \%$ sodium chloride, $0.3 \%$ Triton $\mathrm{X}-100$, and $0.5 \%$ bovine serum albumin. All secondary antisera (Jackson ImmunoResearch, West Grove, PA) were diluted 1:200, raised in donkey, designated for multiple labeling studies, and had minimal cross-reactivity to other relevant species.

Direct immunofluorescence methods were used to detect CRF immunoreactivity and tryptophan hydroxylase (TPH) immunoreactivity. An affinity-purified anti-TPH polyclonal antibody raised in sheep (Chemicon International; Temecula, CA) was used at a dilution of $1: 1000$. The immunolabeling produced with this antisera correlates well with the distribution of serotonergic neurons in the DR. Both CRF and TPH antisera were diluted together and incubated with the tissue for $48 \mathrm{~h}$ at $4{ }^{\circ} \mathrm{C}$ with constant mild agitation. CRF was detected with a rhodamine-conjugated antisera, while TPH was detected using an AMCA-conjugated antisera.

For visualization of NK1 immunolabeling with both CRF and TPH immunolabeling, a rabbit antisera to NK1 receptor raised against a C-terminal portion of the rat NK1 receptor (Vigna et al, 1989) was used at dilution of $1: 1000$ (Novus Biologicals, Littleton, CO). The NK1 receptor antisera yields labeling that is abolished by preadsorbtion with the immunogenic peptide and is consistent with the pattern of NK1 receptor gene expression (Vigna et al, 1989; Maeno et al, 1993). Since the CRF and NK1 receptor primary antisera are both raised in rabbit, tissue was processed sequentially. Sections were first processed for dual labeling of NK1 and TPH by incubating in a solution containing both primary antisera for $48 \mathrm{~h}$. To detect NK1 receptor labeling, tissue was sequentially incubated in a biotin-conjugated donkey antirabbit IgG, Vectastain ABC kit reagents (Vector Laboratories) and NEN tyramide kit (NEN Life Science Products, Inc., Boston, MA) using an FITC-conjugated substrate. TPH was detected using an AMCA-conjugated secondary antisera as described above. The tissue was then rinsed and incubated with the CRF antisera for $48 \mathrm{~h}$ at $4^{\circ} \mathrm{C}$. CRF was detected using a rhodamine-conjugated secondary antisera. Cross-recognition of CRF and NK1 by secondary antisera was not apparent.

For immunoperoxidase labeling of CRF, sections were incubated in the CRF antisera $(1: 8000$; CRF antisera raised in rabbit, lot $\mathrm{C70}$, Dr Wylie Vale, The Salk Institute, San Diego, CA) for $48 \mathrm{~h}$ at $4^{\circ} \mathrm{C}$. Sections were rinsed and incubated in biotinylated donkey anti-rabbit antisera for $90 \mathrm{~min}$ at room temperature. The sections were then rinsed and incubated with avidin-biotin complex (ABC kit, Vector Laboratories) for $90 \mathrm{~min}$. Sections were rinsed and immersed in $0.02 \% 3,3^{\prime}$-diaminobenzidine-4HCl (DAB; Sigma) containing $0.01 \% \mathrm{H}_{2} \mathrm{O}_{2}$ in $\mathrm{PB}$. The $\mathrm{DAB}$ reaction was terminated by rinses in $\mathrm{PB}$.

Immunolabeling of Pha- $\mathrm{L}$ was visualized using antisera raised in goat (1:20000; Vector Laboratories). For immunohistochemical detection of Pha-L alone, the antiserum was diluted 1:20000 and detected using the ABC kit with 
the substrate DAB. For dual labeling of Pha-L and CRF, two methods were used. For brightfield microscopy, sections were sequentially processed first for visualization of CRF using the $\mathrm{ABC}$ kit and the substrate $\mathrm{DAB}$. Immunoperoxidase activity was then quenched by incubating the tissue in $1 \%$ hydrogen peroxide for $30 \mathrm{~min}$. The tissue was then incubated in the antisera for Pha-L, which was detected using the $A B C$ kit and $\mathrm{DAB}$ with the addition of $0.12 \mathrm{~g}$ in $20 \mathrm{ml}$ nickelous ammonium sulfate to yield purple/black fibers. To determine if Pha-L-labeled fibers were dually labeled for CRF, a combined immunoperoxidase-fluorescence method was used. CRF was detected using the $A B C$ kit and $\mathrm{DAB}$, while Pha-L was visualized using a direct FITCconjugated anti-goat IgG.

\section{Figure Preparation}

For black and white photomicroscopy, images were captured using a black and white digital camera and Open Lab software package (Improvision Technology, Coventry, England). For all fluorescence microscopy, black and white images where each fluorophore was illuminated individually were obtained sequentially, pseudocolored and superimposed using Open Lab software. For color bright-field microscopy, images were captured using a color digital camera. Using Adobe Photoshop figures were assembled and adjusted for brightness and contrast. On some panels, sharpness was adjusted using the 'unsharpen mask' filter at $50 \%$ with a 2-4 pixel diameter. Dust grains were removed from several images using the 'rubber stamp' tool.

\section{Semi-Quantitative Analysis}

To estimate the number of CRF-immunoreactive cells that were dually labeled for TPH, at least three sections from each of the three different animals were photographed. Sections were selected randomly from those with good immunohistochemical labeling where an accumulation of CRF-immunoreactive cells was visible. Three images were compared to determine colocalization. First, individual CRF-immunoreactive cells were counted from an image illuminated to visualize only CRF. Using an image of the same field illuminated to visualize single labeling for TPH, and an image of the two fluorophores pseudocolored and merged, the dually labeled cells were counted and a fraction calculated. The fraction of serotonin cells that contained CRF was not determined because TPH-labeled cells were packed tightly together and difficult to clearly enumerate.

Although subject to methodological limitations, the fraction of CRF-immunoreactive cells in the central nucleus of the amygdala that were potentially contacted by Pha- $\mathrm{L}$ labeled fibers was estimated. For this two sections with the best dual-immunocytochemical labeling (blue-brown brightfield visualization) for CRF and Pha-L were used. Individual CRF-labeled cells were counted. Each CRFlabeled cell was then examined for proximity to a fiber with Pha-L immunolabeling. Technical issues severely impact on the ability to detect the colocalization of Pha- $\mathrm{L}$ and CRF, which would result in a substantial number of false-negative results, therefore these observations were not subjected to quantitative analysis.

\section{RESULTS}

\section{CRF and Serotonin Immunolabeling in the DR of Colchicine-Treated Rats}

In tissues from untreated rats, CRF immunoreactivity in the DR was present primarily in axon terminals, which were topographically distributed (Valentino et al, 2001). In contrast, in tissue from colchicine-treated rats, CRF immunolabeling was apparent in a number of neurons in the DR, as well as in axon terminals. To determine whether the CRF-immunoreactive neurons were also serotonergic, tissue from colchicine-treated rats was dually immunolabeled for CRF and TPH (the rate-limiting enzyme involved in serotonin synthesis; Figures 1 and 2). Although absent at the most caudal pole of the DR, at the level of B6 (Molliver, 1987), at mid-rostrocaudal levels, many neurons exhibiting immunolabeling for CRF were visible. These were densely clustered in the dorsomedial subregion and scattered within the lateral wings (Figure 1). Notably, CRF immunoreactive neurons were largely absent from the ventromedial subregion at mid-rostrocaudal levels where the greatest accumulation of serotonergic neurons is located. At more rostral levels of the DR (where the lateral wings are not apparent), CRF-immunoreactive neurons were also observed and were primarily located in the dorsomedial part of the DR (Figure 2). In sections randomly selected from three different rats where the dorsomedial cluster of serotonin neurons was visible, 255/266 (96\%) of cells containing CRF in the dorsomedial DR were dually labeled for $\mathrm{TPH}$.

\section{NK1 Receptor Immunolabeling with Respect to CRF and Serotonin in the DR}

Previously, we and others reported the distribution of NK1 receptor in the DR of the rat (Commons and Valentino, 2002) and mouse (Santarelli et al, 2001). In both species, dendrites with NK1 receptor immunoreactivity were found to selectively surround the dorsomedial DR. Therefore, in this study the distribution of NK1 receptor with respect to CRF-containing cells in the DR was examined. Consistent with previous findings that $\mathrm{NK} 1$ receptor is present in nonserotonin-containing cells and dendrites in the DR (Commons and Valentino, 2002; Santarelli et al, 2001), NK1 receptor immunoreactivity was not colocalized with CRF or TPH immunoreactivity (Figure 3). Rather, dendrites labeled with NK1 receptor radiated in a striking pattern around $\mathrm{CRF} /$ serotonin cells in the dorsomedial part of the DR (Figure 3). The combination of CRF and NK1 receptor immunoreactivity appeared to clearly define the dorsomedial part of the DR.

\section{Targets of CRF-Containing Neurons in the Dorsomedial DR}

To gain further insight into the neural circuits that this part of the DR may functionally modulate, the anterograde tract tracer, Pha-L, was injected into the dorsomedial DR. Figure 4a shows neurons labeled by a small injection that was centered on the midline and just ventral to the aqueduct. This injection site encompassed the dorsal part of the DR and included a few neighboring cells located laterally. 

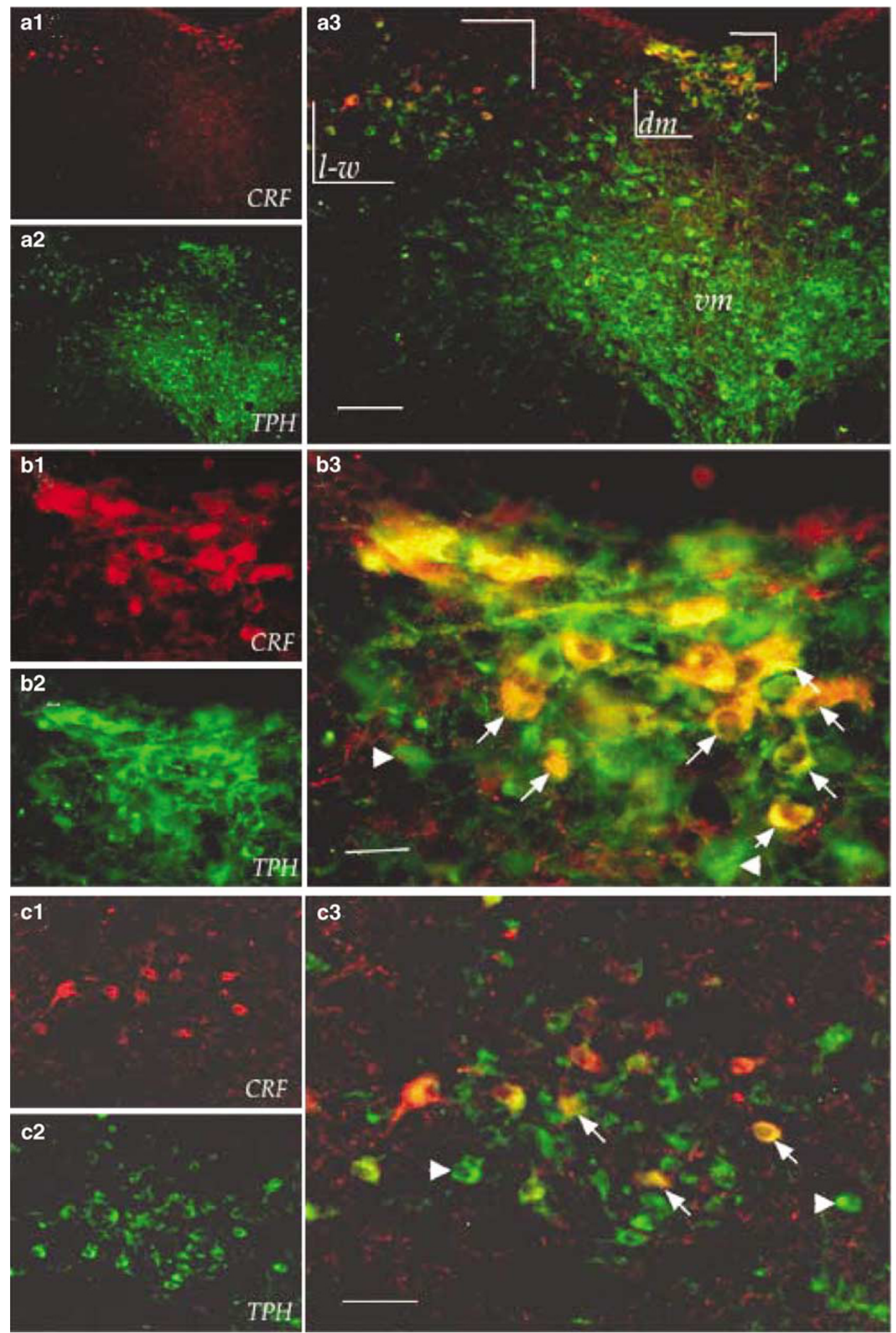

Figure I CRF-immunoreactive cell bodies in the dorsomedial DR and lateral wings contain the serotonin-synthesizing enzyme, TPH. Immunolabeling for CRF (al, red) and TPH (a2, green) at mid-rostrocaudal level through the DR. (a3) Overlay of dual immunolabeling shows that CRF cells in the dorsomedial $(\mathrm{dm})$, as well as those in the lateral wings (I-w) also contain TPH (dual labeling indicated by yellow or orange), whereas CRF immunoreactivity is sparse in the ventromedial $(\mathrm{vm})$ subregion of the DR. Bar $=120 \mu \mathrm{m}$. (b l-3) and $(\mathrm{cl}-3)$ depict bracketed regions in a3 at higher magnification. (bl) Cluster of CRFimmunolabeled cells (red), (b2) TPH (green), and (b3) merged image of the dorsomedial DR reveal that almost all CRF-immunoreactive cells are dually labeled (yellow, some indicated by arrows), apparently comprising a large fraction of serotonin cells in this subregion. Some neurons that are single labeled for TPH are in the field (arrowheads) Bar $=45 \mu \mathrm{m}$. (cl) CRF (red), (c2) TPH (green), and (c3) merged image of the lateral wing show several dually labeled cells (arrows) interspersed with cells single labeled for TPH (arrowheads). Bar $=60 \mu \mathrm{m}$. 

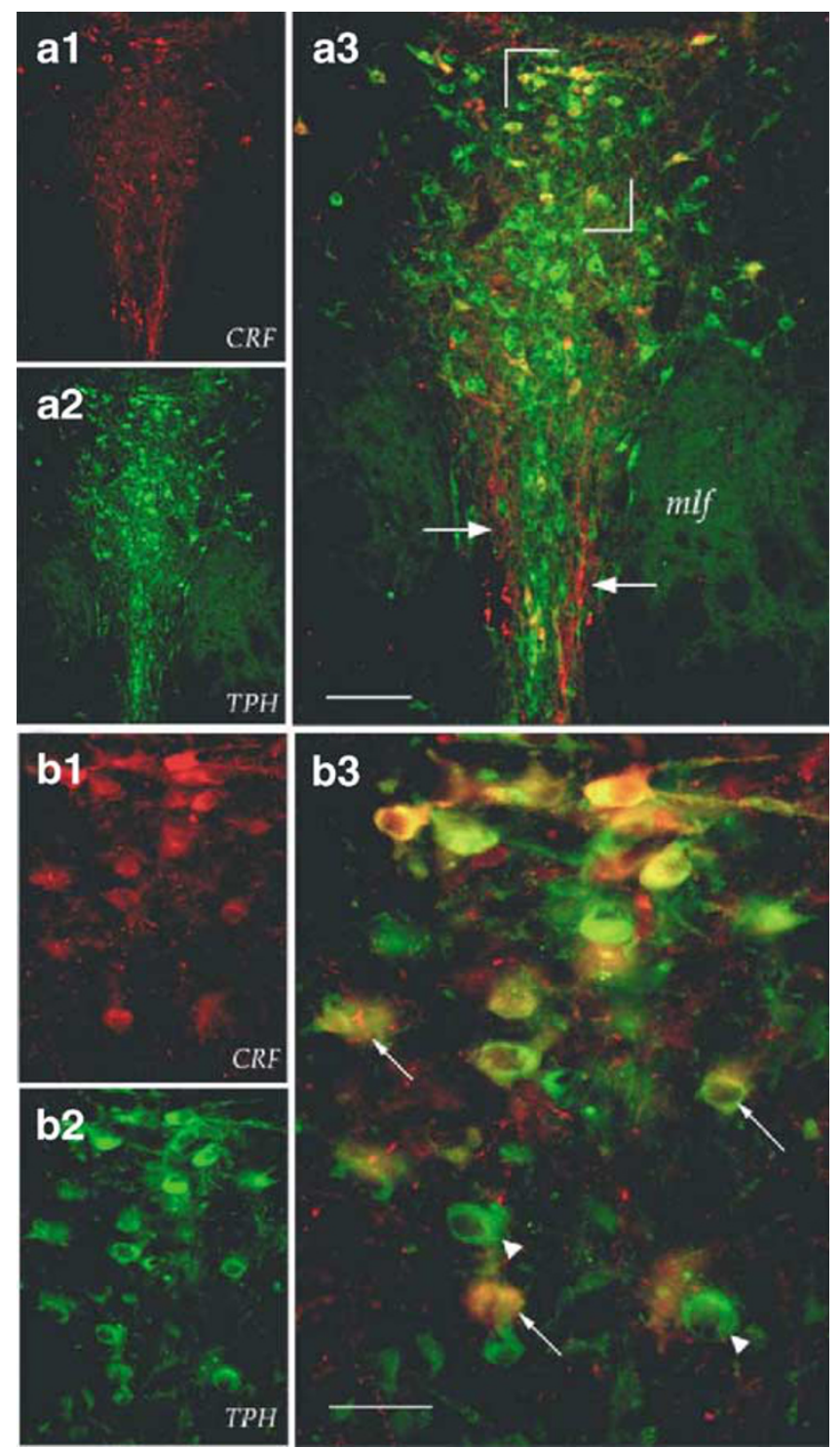

Figure 2 CRF-immunoreactive cell bodies in the dorsal region of the rostral DR also contain TPH. (al) CRF (red), (a2) TPH (green), and (a3) merged image showing that CRF-immunolabeled cell bodies are dually labeled for TPH (yellow) and are most numerous dorsally, whereas CRFimmunoreactive fibers are ventromedial (arrows) in the interfasicular region. $\mathrm{mlf}$, medial longitudinal fasiculus. Bar $=135 \mu \mathrm{m}$. (b I) CRF (red), (b2) TPH (green), and (b3) merged higher magnification of bracketed area in (a3). Cells dually labeled for CRF and TPH (arrows, merged arrows) are interspersed with cells singly labeled for TPH (arrowhead). Bar $=45 \mu \mathrm{m}$.

However, ventral portions of the DR as well as the lateral wing area were spared. This resulted in well-defined, discrete anterograde-labeling pattern. Fibers containing Pha-L were relatively sparse, consisting of only individual fibers in several areas that are major targets of the DR including all cortical regions, hippocampus, caudate putamen, septum, all thalamic areas and the ventral medulla. However, anterogradely labeled fibers densely innervated selective regions, which included the central nucleus of the amygdala (Figure 4b), dorsal hypothalamic area (Figure 4c), and the bed nucleus of the stria terminalis (Figure $4 \mathrm{~d}$ ). Within the amgydala, Pha-L-containing fibers appeared to selectively target the central nucleus of the amygdala, largely sparing the basolateral amygdala as well as other subregions (Figure $4 \mathrm{~b}$ ). The highest density of Pha-L-labeled fibers were found in the lateral and medial parts of the central nucleus of the amygdala, while only few fibers were visible in the capsular portion of this nucleus. In the bed nucleus of the stria terminalis, anterogradely labeled fibers were visible in both the dorsal and ventral parts of the lateral division. An intermediate density of fibers was present in the rostral (but not caudal) pole of the locus coeruleus and Barrington's nucleus.

Owing to the selective distribution of Pha-L-labeled fibers in the lateral part of central nucleus of the amygdala where a major cluster of CRF-containing neurons is located, tissue that was dual labeled for CRF and Pha-L was examined. Pha-L-labeled fibers exhibited a pronounced overlap with CRF cells in the central nucleus of the amygdala (Figure 5a). Of visible CRF-immunoreactive cell bodies many (54\%; 57/ 104) were surrounded by a Pha-L-labeled fibers (Figure $5 b$ and $\mathrm{c}$ ).

Combining immunoperoxidase labeling for CRF to maximize detection and immunofluorescence detection of Pha-L, the presence of CRF within fibers anterogradely labeled from the DR was examined (Figure 6). In addition to CRF cells in the central nucleus of the amygdala, many punctate or varicose fibers, consistent with axonal boutons or axonal fibers, were immunolabeled with CRF, as observed with bright-field microscopy. In some cases, these punctate CRF-immunolabeled structures were in close proximity with the CRF-immunolabeled cell bodies (Figure $6 \mathrm{a} 1$ and $\mathrm{b} 1)$. Although technically subject to underdetection, several examples were noted where CRF-immunolabeled fibers surrounding CRF-containing neurons in the central nucleus of the amygdala were also anterogradely labeled from a Pha-L injection into the dorsomedial DR (Figure 6). These observations suggest that not only do Pha-L-containing axons originating in the dorsal part of the DR surround CRF cells in the central nucleus of the amygdala, but that they also contain CRF.

\section{DISCUSSION}

The present study provides evidence for colocalization of CRF with serotonin in a selective population of neurons in the DR. CRF-serotonin coexistence was most striking in the dorsomedial subregion of the DR. This area is also unique in that this region contains the highest density of NK1 receptor immunolabeling of the entire DR. Electron microscopy has shown that NK1 receptor in this area is present primarily on thin dendritic processes (Commons and Valentino, 2002). Dendrites expressing NK1 receptor immunoreactivity were distinct from, but in close proximity to, serotonin neurons in the dorsomedial DR, as previously reported in rat (Commons and Valentino, 2002) and mouse (Santarelli et al, 2001). Thus, NK1 receptor-expressing neurons may interact with $\mathrm{CRF}$ /serotonin neurons in the dorsomedial DR. Anterograde tract tracing combined with CRF immunohistochemistry revealed that the CRF-containing neurons in the dorsomedial DR target limbic sources of CRF, particularly, CRF-containing neurons of the central nucleus of the amygdala, a structure that has been 

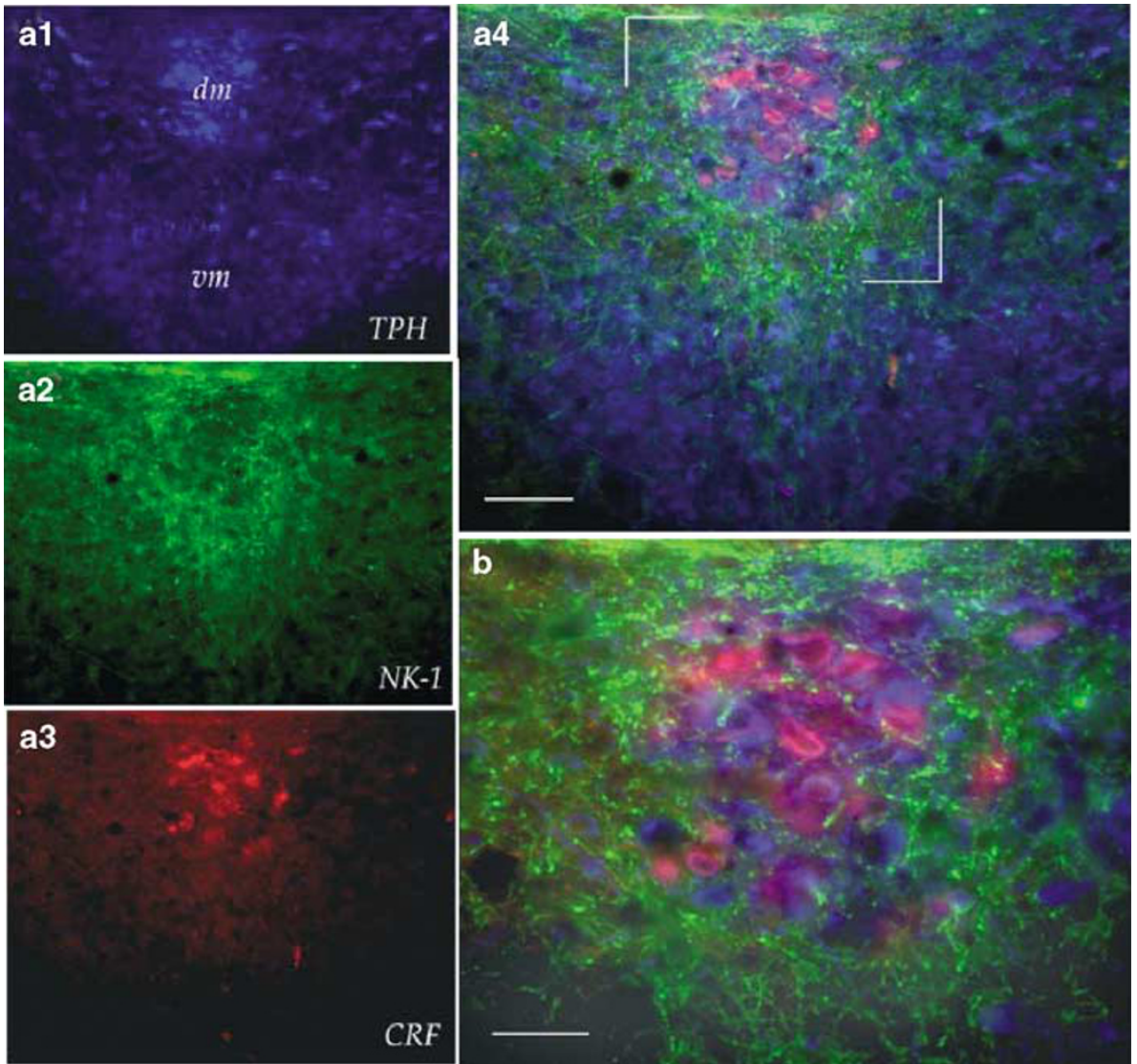

Figure $3 \mathrm{NKI}$ receptor and CRF immunolabeling define the dorsomedial DR. (al) Labeling for TPH (blue) reveals dorsomedial (dm) and ventromedial (vm) subregions of the DR. (a2) Immunolabeling for NKI receptor, primarily associated with thin dendrites (green) and (a3) CRF (red). (a4) Merged images of panels (al-3): CRF-immunoreactive cells clustered in the dorsomedial DR are dually labeled for TPH (visualized as pink-purple) and are surrounded by thin dendritic processes labeled for NKI receptor (green). NKI receptor immunolabeling does not colocalize with either TPH or CRF, but represents a distinct population of cells. Bar $=120 \mu \mathrm{m}$. (b) Higher magnification of bracketed region in (a) shows that CRF-TPH dually labeled cells (pink-purple) and NKI receptor (green) labeled processes are distinct. Bar $=60 \mu \mathrm{m}$.

implicated in the behavioral expression of fear (LeDoux et al, 1988). Taken together, the present findings suggest the existence of a circuit whereby NK1 receptor activation in the dorsomedial DR can impact limbic sources of CRF via its effects on $\mathrm{CRF} /$ serotonin neurons in the dorsomedial DR. By converging on this common circuit at different points, diverse pharmacological agents (eg NK1 receptor antagonists, CRF antagonists, and serotonin reuptake inhibitors) may have overlapping clinical consequences.

\section{Technical Consideration}

As is often the case for optimal visualization of peptide immunoreactivity in somatodendritic processes, colchicine pretreatment was necessary for visualization of CRF immunolabeling in somatodendritic processes of the DR. As a result of this, it might be argued that DR neurons do not express CRF under normal conditions. However, occasionally light somatodendritic immunolabeling for CRF could be detected in the dorsomedial DR under normal conditions. Moreover, some fibers anterogradely labeled from the dorsomedial DR were CRF immunoreactive in noncolchicine-treated rats. The possibility that stress or other conditions regulate CRF expression in this cell population is intriguing and remains to be investigated.

A caveat of anterograde tract tracing in this study is that it is not possible to selectively label the CRF/serotonin neurons in the dorsomedial DR. Thus, the anterograde labeling that was observed may represent projections from other neurons in the vicinity. This may be the case with anterograde labeling in the bed nucleus of the stria terminalis because previous retrograde tract tracing studies from this area have suggested that periaqueductal neurons adjacent to CRF/serotonin cell clusters project to this region (Kozicz et al, 1998). In contrast, the conclusion that the dorsomedial DR projects to the central nucleus of the amygdala and hypothalamus is consistent with retrograde tracing studies (Ottersen, 1981; Uryu et al, 1992; Champagne et al, 1998). In fact, hypothalamic afferents from the DR were previously reported to contain CRF (Champagne et al, 1998), and although technically difficult to accurately quantify, CRF immunolabeling was found in anterogradely labeled fibers in the amygdala (this study). Finally, it is noteworthy that anterogradely labeled fibers were sparse in other brain regions that typically exhibit substantial labeling from larger Pha-L injections into the DR (Vertes, 1991; Vertes and Kocsis, 1994), suggesting that injections 

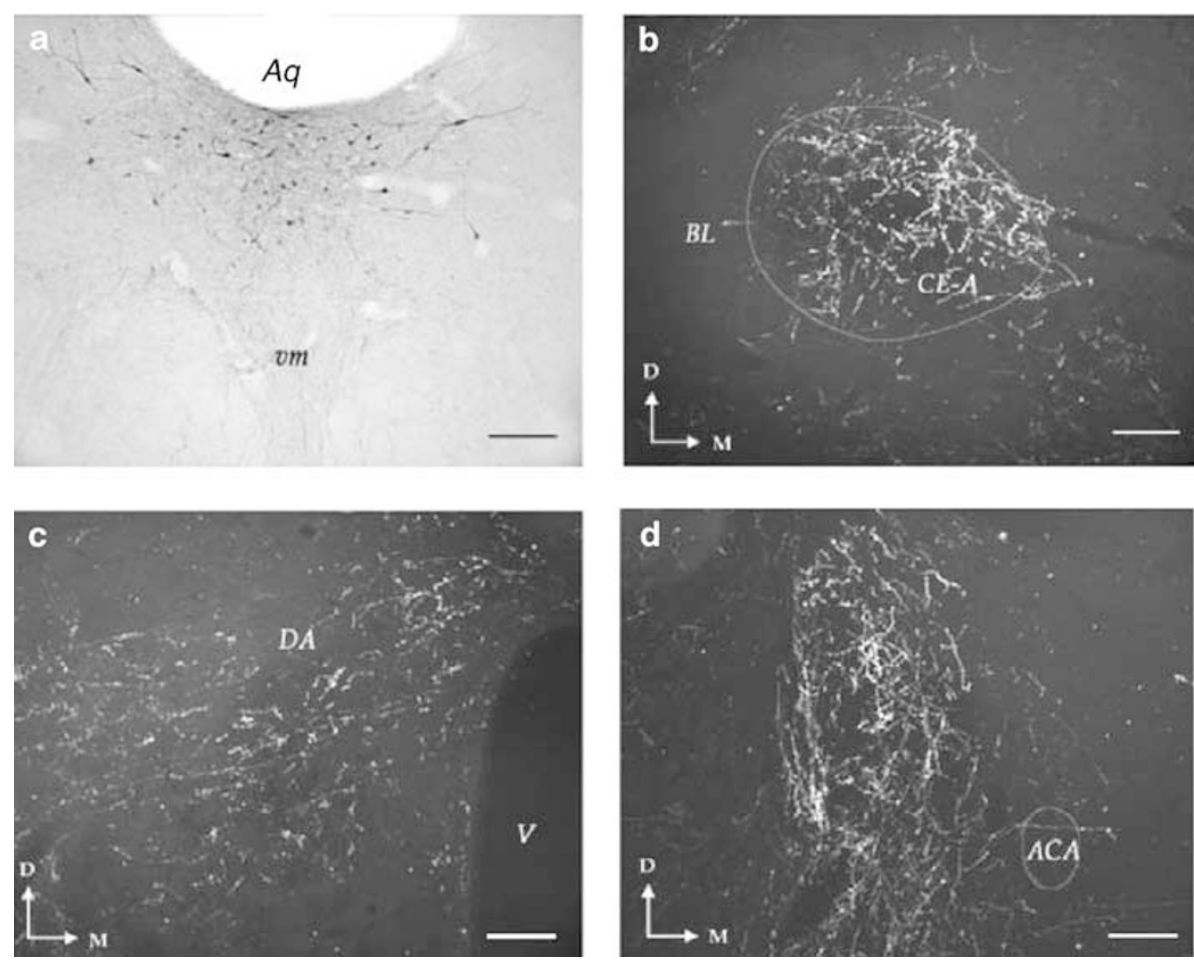

Figure 4 Projection areas of the dorsal DR. (a) Injection site of the anterograde tract tracer Pha-L into the dorsal DR is centered on the midline just below the aqueduct ( $\mathrm{Aq}$ ) and spreads laterally, but has limited incursion into the vm portion of the DR. (b) Pha-L-labeled fibers are numerous in the central nucleus of the amygdala (CE-A) while other amygdalar regions for example the basolateral (BL), are largely spared. (c) Pha- $L$ visualized in the dorsal hypothalamic area $(\mathrm{DA})$ lateral to the apex of the ventricle $(\mathrm{V})$. (d) Dense innervation was also present in the bed nucleus of the stria terminalis lateral to the anterior commisure (ACA). Dorsal (D) and medial (M) orientation indicated by the arrows in each panel. Bars $=235 \mu \mathrm{m}$.
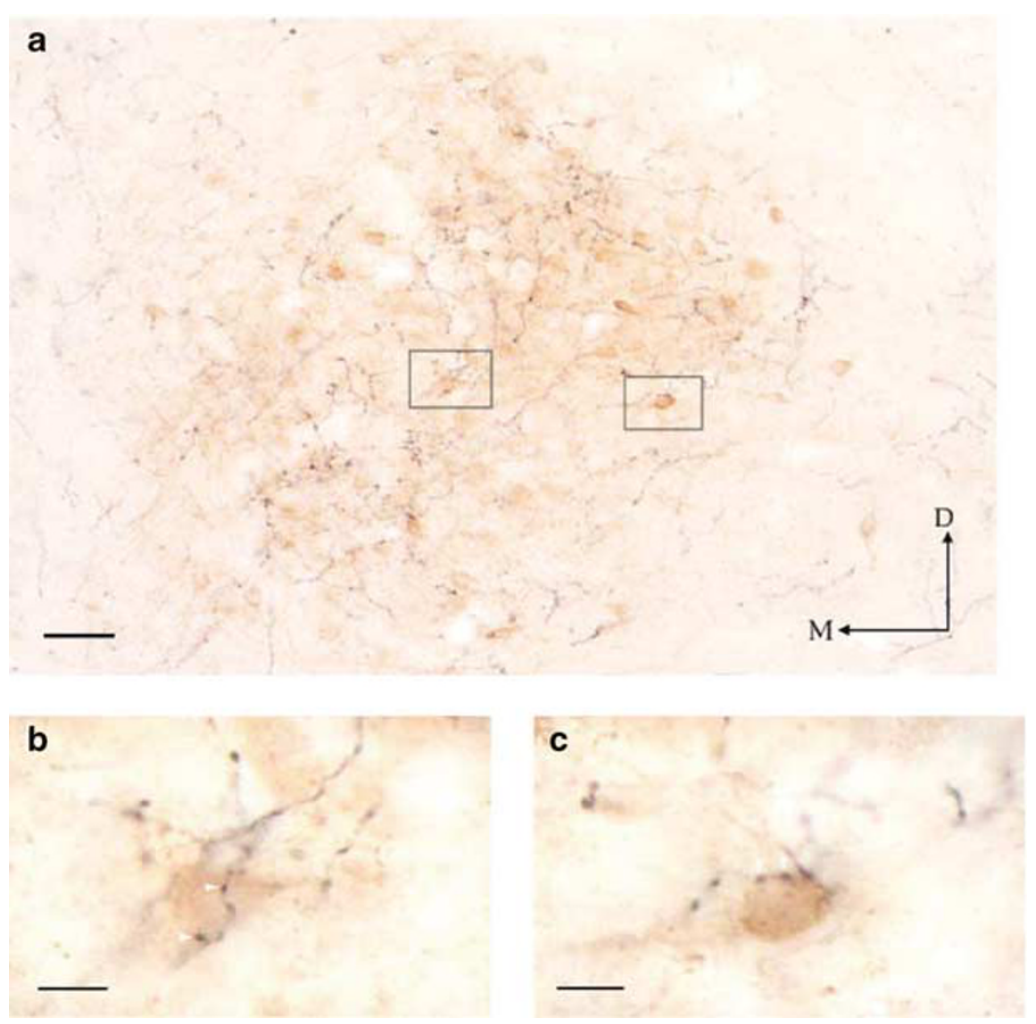

Figure 5 Projections from the dorsal DR target CRF neurons in the central nucleus of the amygdala. (a) Area of the central nucleus of the amygdala rich in CRF (visualized brown) appears selectively targeted by fibers from the dorsal DR labeled with Pha- $L$ (visualized blue-black, injection site shown in Figure $5 a$ ) Dorsal (D) and medial (M) orientation indicated by arrows. Bar $=120 \mu \mathrm{m}$. (b and c) High magnification of boxed regions in (a) showing CRF-immunolabeled neurons (brown) surrounded by Pha-L-labeled fibers suggestive of axo-somatic innervation (arrowheads) by these afferents. Bar $=25 \mu \mathrm{m}$. 


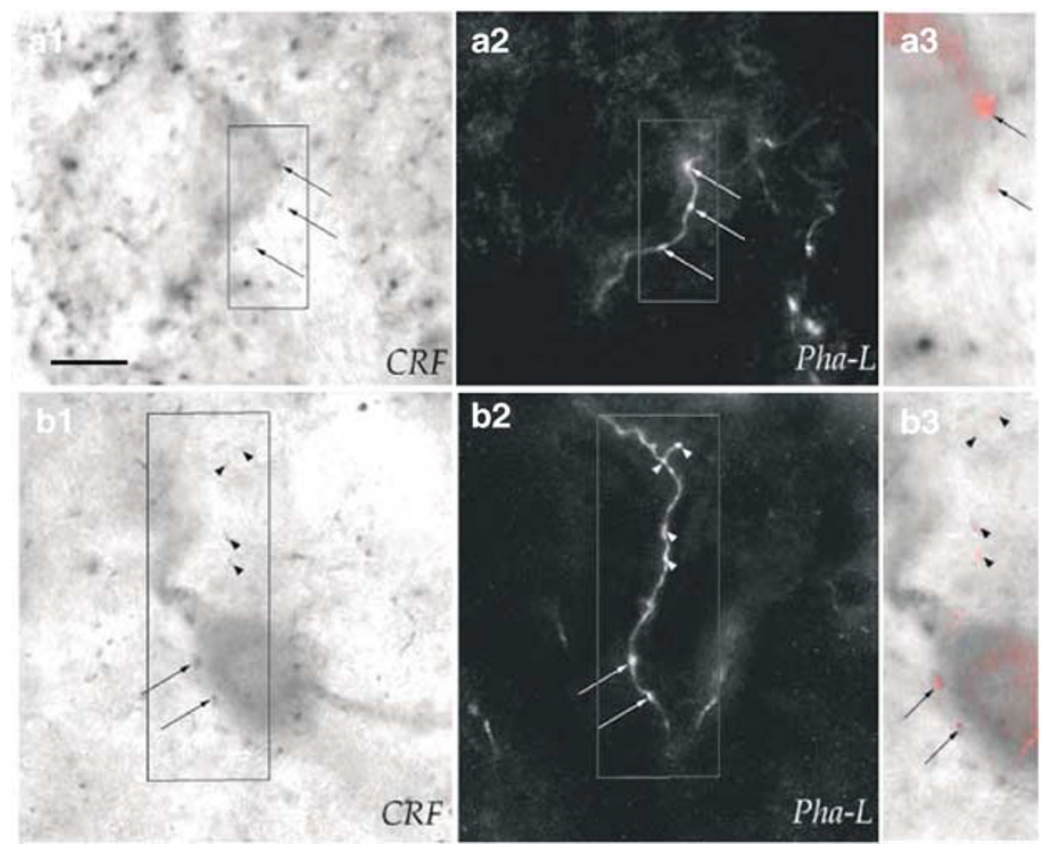

Figure 6 Axons from the dorsomedial DR contained CRF and surround CRF-labeled cells in the central nucleus of the amygdala. (al and bI) To maximize visualization, CRF was detected using the peroxidase-DAB method. Bar $=17 \mu \mathrm{m}$. (a2 and b2) Pha-L-containing fibers labeled using immunofluorescence. Arrows in panels ( $a$ and $b$ ) are in the same register. ( $a 3$ and b3) Merged images of the boxed regions where Pha- $L$ is colorized red. Boutons dually labeled for CRF and Pha-L surround cells labeled for CRF (arrows). (b3) CRF immunoreactivity is also in exact register with portions of the Pha-L-labeled axon distal to the CRF-labeled cell body (arrowheads).

yielding the labeling shown in this study were relatively discrete.

\section{Potential Functions of CRF in Serotonin Neurons}

CRF that is colocalized with serotonin in DR neurons could act through local circuits to regulate activity of nearby DR neurons or local serotonin release. CRF receptor binding sites and mRNA are abundant in the DR (Rominger et al, 1998). Ultrastructural studies in rats not treated with colchicine demonstrated that CRF terminals form synaptic specializations with dendrites and are also apposed to nonlabeled terminals in the dorsal region of the caudal DR (Valentino et al, 2001). Although it is likely that many of the CRF terminals in this region derive from outside the DR, it is possible that CRF-immunoreactive neurons identified in the DR have local axon collaterals. Local application of relatively low doses of CRF inhibits DR neuronal activity in vivo (Kirby et al, 2000). Although CRF-induced excitation of DR neuronal activity has been reported in vitro, these effects were localized to more ventral regions of the caudal DR (Lowry et al, 2000). Thus, CRF release from neurons within the DR may function in part to provide a feedback inhibition of serotonergic neurons in the DR.

Anterograde tracing from the dorsomedial DR suggested that this subnucleus targets CRF-containing brain nuclei (eg central nucleus of the amygdala, dorsal hypothalamic area), and retrograde tracing studies are consistent with this (Champagne et al, 1998; Uryu et al, 1992). Moreover, evidence for collateralization of serotonin projections from the caudal DR to the amygdala and hypothalamus has been reported (Petrov et al, 1994). In the central nucleus of the amygdala, some anterogradely labeled fibers were CRF immunoreactive and appeared to specifically target the CRF-containing neurons. CRF could function presynaptically in this pathway to regulate serotonin release in the central nucleus of the amygdala. In this regard, a number of stressors alter serotonin release in the amygdala (Kirby et al, 1995, 1997) and this may be presynaptically modulated by CRF. Alternatively, CRF from the dorsomedial DR could act as a co-neurotransmitter with serotonin to have postsynaptic effects on CRF-containing amygdala neurons. CRF inhibits activity of neurons of the central nucleus of the amygdala in vitro, although it is not known whether the amygdalar neurons that are receptive to CRF are also CRF containing (Rainnie et al, 1992). These observations suggest that DR-CRF may serve to feedback regulate amygdalar CRF neurons during stress.

\section{Dorsomedial DR as a Point of Intersection of CRF, Serotonin, and NK1}

Given that the central nucleus of the amygdala is a key component of circuits underlying emotion (LeDoux, 1988), it has a potential role in the pathophysiology of affective disorders, including anxiety and depression. The CRF output of the amygdala is thought to play a pivotal role in mediating the behavioral responses to fearful and/or stressful situations (Fendt et al, 1997; Koob, 1999; Liang et al, 1992; Shepard et al, 2000). The present findings suggest a circuit through which NK1 receptor antagonists, CRF antagonists, and serotonin reuptake inhibitors may converge to produce the common consequence of inhibition of CRF-amygdalar output. The well-defined localization of NK1 receptors in the dorsomedial DR suggests that ligands of this receptor may act on nonserotonergic populations to 
impact the network activity preferentially within this subregion. Here, NK1 receptive processes can interact with $\mathrm{CRF} /$ serotonin projections, which in turn modulate CRF neurons in the central nucleus of the amygdala. Although the precise actions of NK1 receptor agonists on serotonergic activity remains speculative, the finding that DR neuronal activity is increased in transgenic mice lacking NK1 receptors, or in wild-type mice administered NK1 antagonists, suggests that NK1 receptor activation is inhibitory to DR neurons (Santarelli et al, 2001). Interestingly, these manipulations are associated with behavioral indices suggesting an anxiolytic effect (Santarelli et al, 2001).

The effects of serotonin administration or DR stimulation on amygdalar neurons are generally inhibitory through activation of $5-\mathrm{HT}_{1 \mathrm{~A}}$ receptors (Wang and Aghajanian, 1977). Therefore, inhibition of serotonergic activity via NK1 receptor activation in the dorsomedial DR could act to release CRF neurons in the central nucleus of the amygdala from serotonergic inhibition. Conversely, NK1 receptor antagonists would be predicted to increase inhibitory regulation of CRF-containing amygdala neurons by the dorsomedial DR and this could contribute to anxiolytic effects.

Novel anxiolytics that are CRF receptor antagonists may act at multiple sites within this proposed circuit. We and others have provided evidence that CRF participates in modulating DR function as a consequence of stress (Hammack et al, 2002; Kirby et al, 2000; Lowry et al, 2000). In the present study, novel evidence is presented that $\mathrm{CRF}$ may function as a co-neurotransmitter with serotonin, and this may occur selectively within the central nucleus of the amygdala. Finally, CRF antagonists would be predicted to block the effects of CRF-amygdala neurons in their projection areas, that is, at the final limb of the circuit. Therefore, the putative circuit suggested by the present findings may be pharmacologically manipulated at multiple sites by diverse pharmacological agents that are effective in affective disorders. Inhibiting the CRF output from the central nucleus of the amygdala may be the common denominator for efficacy of ligands that modify NK1, CRF, and serotonergic neurotransmission in affective disorders. As these agents act at different sites within the circuit, combined pharmacotherapy to individualize and optimize efficacy and minimize side effects is an intriguing prospect.

\section{ACKNOWLEDGMENTS}

This work was supported by PHS Grants DA-14329, DA-00463 (a Mentored Research Scientist Development Award to KGC), MH 58250, and MH 02006 (Research Scientist Award to RJV). The technical expertise of Mr Vincent Bey is gratefully acknowledged.

\section{REFERENCES}

Arango V, Underwood MD, Boldrini M, Tamir H, Kassir SA, Hsiung $S$ et al (2001). Serotonin 1A receptors and serotonin transporter mRNA expression in the brainstem of depressed suicide victims. Neuropsychopharmacology 25: 892-903.

Argyropoulos SV, Nutt DJ (2000). Substance P antagonists: novel agents in the treatment of depression. Expert Opin Invest Drugs 9: $1871-1875$.
Champagne D, Beaulieu J, Drolet G (1998). CRFergic innervation of the paraventricular nucleus of the rat hypothalamus: a tracttracing study. J Neuroendocrinol 10: 119-131.

Commons K, Valentino RJ (2002). Cellular basis for the effects of Substance $\mathrm{P}$ in the periaqueductal gray and dorsal raphe nucleus. J Comp Neurol 447: 82-97.

Cowen PJ (1993). Serotonin receptor subtypes in depression: evidence from studies in neuroendocrine regulation. Clin Neuropharmacol 16(Suppl 3): S6-S18.

Fendt M, Koch M, Schnitzler HU (1997). Corticotropin-releasing factor in the caudal pontine reticular nucleus mediates the expression of fear-potentiated startle in the rat. Eur J Neurosci 9: 299-305.

Hammack SE, Richey KJ, Schmid MJ, LoPresti ML, Watkins LR, Maier SF (2002). The role of corticotropin-releasing hormone in the dorsal raphe nucleus in mediating the behavioral consequences of uncontrollable stress. J Neurosci 22: 1020-1026.

Jacobs BL, Azmitia EC (1992). Structure and function of the brain serotonin system. Physiol Rev 72: 165-228.

Kirby LG, Allen AR, Lucki I (1995). Regional differences in the effects of forced swimming on extracellular levels of 5hydroxytryptamine and 5-hydroxyindoleacetic acid. Brain Res 682: 189-196.

Kirby LG, Chou-Green JM, Davis K, Lucki I (1997). The effects of different stressors on extracellular 5-hydroxytryptamine and 5hydroxyindoleacetic acid. Brain Res 760: 218-230.

Kirby LG, Rice K, Valentino RJ (2000). Effects of corticotropinreleasing factor on neuronal activity in the serotonergic dorsal raphe nucleus. Neuropsychopharmacology 22: 148-162.

Koob GF (1999). Corticotropin-releasing factor, norepinephrine, and stress. Biol Psychiatry 46: 1167-1180.

Kozicz T, Yanaihara H, Arimura A (1998). Distribution of urocortin-like immunoreactivity in the central nervous system of the rat. J Comp Neurol 391: 1-10.

LeDoux JE, Iwata J, Cicchetti P, Reis DJ (1988). Different projections of the central amygdaloid nucleus mediate autonomic and behavioral correlates of conditioned fear. J Neurosci 8: 2517-2529.

Lesch KP (1991). 5-HT1A receptor responsivity in anxiety disorders and depression. Prog Neuropsychopharmacol Biol Psychiatry 15: 723-733.

Liang KC, Melia KR, Campeau S, Falls WA, Miserendino MJ, Davis $M$ (1992). Lesions of the central nucleus of the amygdala, but not the paraventricular nucleus of the hypothalamus, block the excitatory effects of corticotropin-releasing factor on the acoustic startle reflex. J Neurosci 12: 2313-2320.

Lowry CA, Rodda JE, Lightman SL, Ingram CD (2000). Corticotropin-releasing factor increases in vitro firing rates of serotonergic neurons in the dorsal raphe nucleus: evidence for activation of a topographically organized mesolimbocortical serotonergic system. J Neurosci 20: 7728-7736.

Maeno H, Kiyama H, Tohyama M (1993). Distribution of the substance $\mathrm{P}$ receptor (NK-1 receptor) in the central nervous system. Brain Res Mol Brain Res 18: 43-58.

Mann JJ, Malone KM, Diegl DJ, PErel J, Cooper TB, Mintun MA (1996). Demonstration in vivo of reduced serotonin responsivity in the brain of untreated depressed patients. Am J Psychiatry 153: 174-182.

Molliver ME (1987). Serotonergic neuronal systems: what their anatomic organization tells us about function. J Clin Psychopharmacol 7: 3S-23S.

Nordstrom P, Asberg M (1992). Suicide risk and serotonin. Int Clin Psychopharmacol 6(Suppl 6): 6-21.

O'Hearn E, Molliver ME (1984). Organization of raphe-cortical projections in rat: a quantitative retrograde study. Brain Res Bull 13: 709-726.

Petrov T, Krukoff TL, Jhamandas JH (1994). Chemically defined collateral projections from the pons to the central nucleus of the 
amygdala and hypothalamic paraventricular nucleus in the rat. Cell Tissue Res 277: 289-295.

Peyron C, Luppi P-H, Fort P, Rampon C, Jouvet M (1996). Lower brainstem catecholamine afferents to the rat dorsal raphe nucleus. J Comp Neurol 364: 402-413.

Peyron C, Petit J-M, Rampon C, Jouvet M, Luppi P-H (1998). Forebrain afferents to the rat dorsal raphe nucleus demonstrated by retrograde and anterograde tracing methods. Neuroscience 82: 443-468.

Price ML, Lucki I (2001). Regulation of serotonin release in the lateral septum and striatum by corticotropin-releasing factor. $J$ Neurosci 21: 2833-2841.

Rainnie DG, Fernhout BJH, Shinnick-Gallagher P (1992). Differential actions of corticotropin releasing factor on basolateral and centralamygdaloid neurons, in vitro. J Pharmacol Exp Ther 263: 846-858.

Rominger DH, Rominger CM, Fitzgerald LW, Grzanna R, Largent BL, Zaczek R (1998). Characterization of [125I] sauvagine binding to $\mathrm{CRH} 2$ receptors: membrane homogenate and autoradiographic studies. J Pharmacol Exp Ther 286: 459-468.

Ruggiero DA, Underwood MD, Rice PM, Mann JJ, Arango V (1999). Corticotropin-releasing hormone and serotonin interact in the human brainstem: behavioral implications. Neuroscience 91: 1343-1354.

Santarelli L, Gobbi G, Debs PC, Sibille EL, Blier P, Hen R et al (2001). Genetic and pharmacological disruption of neurokinin 1 receptor function decreases anxiety-related behaviors and increases serotonergic function. Proc Natl Acad Sci 98: 1912-1917.

Shapira B, Yagmur MJ, Gropp C, Newman M, Lerer B (1992). Effect of clomipramine and lithium on fenfluramine-induced hormone release in major depression. Biol Psychiatry 31: 975-983.
Shepard JD, Barron KW, Myers DA (2000). Corticosterone delivery to the amygdala increases corticotropin-releasing factor mRNA in the central amygdaloid nucleus and anxiety-like behavior. Brain Res 861: 288-295.

Uryu K, Okumura T, Shibasaki T, Sakanaka M (1992). Fine structure and possible origins of nerve fibers with corticotropinreleasing factor-like immunoreactivity in the rat central amygdaloid nucleus. Brain Res 577: 175-179.

Valentino RJ, Liouterman L, Van Bockstaele EJ (2001). Evidence for regional heterogeneity in corticotropin-releasing factor interactions in the dorsal raphe nucleus. J Comp Neurol 435: 450-463.

van Praag HM (1984). Depression, suicide, and serotonin metabolism in the brain. In: Post RM, Ballenger JC (eds). Neurobiology of Mood Disorders. Williams \& Wilkins: Baltimore, MD. pp 601-618.

Vertes RP (1991). A Pha-L analysis of ascending projections of the dorsal raphe nucleus in the rat. J Comp Neurol 313: 643-668.

Vertes RP, Kocsis B (1994). Projections of the dorsal raphe nucleus to the brainstem: Pha-L analysis in the rat. J Comp Neurol 340: $11-26$.

Vigna SR, Mantyh CR, Soll AH, Maggio JE, Mantyh PW (1989). Substance $\mathrm{P}$ receptors on canine chief cells: localization, characterization, and function. J Neurosci 9: 2878-86.

Wang RY, Aghajanian GK (1977). Inhibition of neurons in the amygdala by dorsal raphe stimulation: mediation through a direct serotonergic pathway. Brain Res 120: 85-102.

Zobel AW, Nickel T, Kunzel HE, Ackl N, Sonntag A, Ising M et al (2000). Effects of the high-affinity corticotropin-releasing hormone receptor 1 antagonist R121919 in major depression: the first 20 patients treated. J Psychiatr Res 34: 171-181. 